\title{
Scale Symmetry Breaking from the Dynamics of Maximal Rank Gauge Field Strengths
}

\author{
E. I. Guendelman ${ }^{1}$ \\ Physics Department, Ben Gurion University, Beer Sheva 84105, Israel
}

\begin{abstract}
Scale invariant theories which contain maximal rank gauge field strengths (of $D$ indices in $D$ dimensions) are studied. The integration of the equations of motion of these gauge fields leads to the s.s.b. of scale invariance. The cases in study are: i) the spontaneous generation of $r^{-1}$ potentials in particle mechanics in a theory that contains only $r^{-2}$ potentials in the scale invariant phase, ii) mass generation in scalar field theories, iii) generation of non trivial dilaton potentials in generally covariant theories, iv) spontaneous generation of confining behavior in gauge theories. The possible origin of these models is discussed.
\end{abstract}

\section{Introduction}

The idea that among the fundamental laws of physics, we have scale invariance (s.i.) as one of the fundamental principles appears as an attractive possibility. In its most naive realizations, such a symmetry is not a viable symmetry, however, since nature seems to have chosen some typical scales, so s.i. has to be broken somehow.

The origin of scales out of an originally scale invariant theory has a long history. In most of the previous research, quantum effects that break s.i. have been investigated ${ }^{1,2,3}$. The breaking of s.i. is crucial in the so called "induced gravity ideas". Here the Planck scale can originate from the s.i. breaking,

\footnotetext{
${ }^{1}$ Electronic address: guendel@bgumail.bgu.ac.il
} 
which can be explicit ${ }^{4}$ (from an assumed scalar field potential which contains a mass parameter) or may have its origin in quantum fluctuations ${ }^{5,6}$.

While one may look at quantum effects for the origin of scale symmetry breaking, there is also a way to achieve this at the classical level in certain models (as we will discuss in the last section, the "classical" scale symmetry breaking effect may be nevertheless related to some infrared singular behavior of an associated quantum theory in some cases).

In the first scale invariant model of this kind ${ }^{7}$, a metric, a dilaton field and a "measure field" $\Phi$ are introduced. The unusual modified measure ${ }^{8}$ is an object that has the same transformation properties under general coordinate transformations as $\sqrt{-g} d^{4} x\left(g=\operatorname{det}\left(g_{\mu \nu}\right)\right)$. $\Phi$ is a density built out of degrees of freedom independent of the metric. For example, given a four index field strength (in four dimensions) $F_{\mu \nu \alpha \beta}=\partial_{[\mu} A_{\nu \alpha \beta]}$, the measure field $\Phi$ is defined as $\Phi=\epsilon^{\mu \nu \alpha \beta} F_{\mu \nu \alpha \beta}$. One may consider the field strength to be composed of elementary scalars ${ }^{9}$. Four in the case of a four index field strength, as it was done in Refs. 7, 8.

The scale symmetry breaking in Ref. 7 comes when considering the integration of the equation of motion of the four index field strength, which introduces an arbitrary scale in the equations of motion.

The modified measure idea has been applied to dynamical generation of string and brane tensions ${ }^{10}$, to cosmological ${ }^{11}$ and to the fermion family problem $^{12}$.

Such kind of "maximal rank" (four index in four space-time dimension) Gauge field strength has a simple dynamics and were introduce in the context of non scale invariant theories by several authors ${ }^{13}$.

Here we want to extend the use of "maximal rank" field strengths for the purpose of s.s.b. of scale invariance in models not involving gravity , including models in i) particle mechanics, where $\frac{1}{r}$ potentials appear after s.s.b. of s.i. given that only $\frac{1}{r^{2}}$ potentials appear in the unbroken phase, ii) Field theory models where non trivial scalar field potentials appear with s.s.b. of s.i. and may be also of internal symmetries, iii) the spontaneous generation of confining behavior in gauge theories. For the sake of completeness, we review also the s.s.b. of s.i. in the generally covariant theory studied in Ref. 7 (section 4). 


\section{A particle mechanics example}

Let us start our discussion by considering what is possibly the simplest system displaying scale invariance, a non relativistic particle subjected to an inverse square potential, i.e.

$$
S=\int L d t, L=\frac{1}{2}\left(\frac{d r}{d t}\right)^{2}-\frac{K}{2 r^{2}}
$$

where $K$ is a constant.

It is then straightforward to verify that the following scale transformation is a symmetry of $(1)$,

$$
r(t) \rightarrow r^{\prime}(t)=\lambda^{-\frac{1}{2}} r(\lambda t)
$$

An equivalent form to (1) is obtained by considering the lagrange multiplier $\omega$ in the new action

$$
S=\int L d t, L=\frac{1}{2}\left(\frac{d r}{d t}\right)^{2}+\frac{1}{2} \omega^{2}-\omega \frac{K^{\frac{1}{2}}}{r}
$$

then s.i. is obtained if in addition to (2) we also transform $\omega$ as in $\omega(t) \rightarrow \omega^{\prime}(t)=\lambda^{\frac{1}{2}} \omega(\lambda t)$ here $\omega(t)$ is treated as a new dynamical variable. The equation of motion obtained from the variation of $\omega(t)$ is

$$
\omega(t)=\frac{K^{\frac{1}{2}}}{r}
$$

replacing back into (3) we obtain the action (1). Replacing $\omega(t)$ back into (3) is a legitimate operation because (3) is a constraint equation. In any case, it is very much obvious that (1) and (3) lead to identical equations of motion.

We now consider a generalization of (1) or (3) which will give the solutions of (1) or (3) as a particular choice of the initial conditions. This involves the consideration of the "maximum rank" field strength, which in one dimension means considering $\omega(t)=\frac{d \sigma}{d t}$ and consider

$$
S=\int L d t, L=\frac{1}{2}\left(\frac{d r}{d t}\right)^{2}+\frac{1}{2}\left(\frac{d \sigma}{d t}\right)^{2}-\frac{d \sigma}{d t} \frac{K^{\frac{1}{2}}}{r}
$$

s.i. is obtained if $r$ transforms as in (2) and $\sigma$ transforms as 


$$
\sigma(t) \rightarrow \sigma^{\prime}(t)=\lambda^{-\frac{1}{2}} \sigma(\lambda t)
$$

Notice the existence of the additional global symmetry

$$
\sigma(t) \rightarrow \sigma(t)+\text { constant }
$$

Now the equation of motion that is obtained from the variation with respect to $\sigma$ is

$$
\frac{d}{d t}\left(\frac{d \sigma}{d t}-\frac{K^{\frac{1}{2}}}{r}\right)=0
$$

which can be integrated to give

$$
\frac{d \sigma}{d t}=\frac{K^{\frac{1}{2}}}{r}+M
$$

where $M$ is an integration constant. It corresponds to the conserved charge associated to the internal symmetry (7). As we will see the constant $M$ leads to s.s.b. of scale symmetry. One may notice the interesting fact that the presence of a non trivial charge can lead to s.s.b ${ }^{14}$. Equation (9) cannot be used in order to solve $\frac{d \sigma}{d t}$ and then replace this value in eq. (5), that procedure leads to incorrect results.

The equation of motion associated with the variation with respect to $r$ (treating $\frac{d \sigma}{d t}$ in (5) as independent of $\mathrm{r}$, as should be done in the correct variational principle) gives

$$
\frac{d^{2} r}{d t^{2}}=-\frac{d \sigma}{d t} \frac{\partial}{\partial r}\left(\frac{K^{\frac{1}{2}}}{r}\right)
$$

and using (9) we get,

$$
\frac{d^{2} r}{d t^{2}}=\frac{K}{r^{3}}+\frac{M K^{\frac{1}{2}}}{r^{2}}=-\frac{\partial}{\partial r} V_{e f f}
$$

where

$$
V_{e f f}=\frac{1}{2} \frac{K}{r^{2}}+\frac{M K^{\frac{1}{2}}}{r}
$$

We see that the constant of integration $M$ leads to a $\frac{1}{r}$ term in the effective potential. Notice that if we had added an explicit $\frac{1}{r}$ term in the action (1), this would have explicitly violated scale invariance. Instead, we have 
now obtained the breaking of scale invariance after the integration of the equations of motion. $M \neq 0$ means indeed s.s.b. of scale invariance.

The spontaneous generation of a $\frac{1}{r}$ term in the effective potential, in a theory where such a potential does not exist in its scale invariant phase, resembles very much the idea of induced gravity ${ }^{4,5,6}$, where the Newtonian gravity (the analogous of a $\frac{1}{r}$ term in the effective potential here) appears as a result of the breaking of s.i..

\section{Scalar Field Theory with "Maximal Rank" Gauge Fields}

We consider first the scale invariant model, formulated in a flat four dimensional space-time:

$$
S=\int L d^{4} x, L=\frac{1}{2} \frac{\partial \phi}{\partial x^{\mu}} \frac{\partial \phi}{\partial x_{\mu}}-\frac{1}{2} \lambda \phi^{4}
$$

Such a model is invariant under the scale transformation

$$
\phi(x) \rightarrow \phi^{\prime}(x)=\lambda \phi(\lambda x)
$$

As in the previous section, we can consider instead of (13) the following equivalent action

$$
S=\int L d^{4} x, L=\frac{1}{2} \frac{\partial \phi}{\partial x^{\mu}} \frac{\partial \phi}{\partial x_{\mu}}+\frac{1}{2} \omega^{2}-\omega \lambda^{\frac{1}{2}} \phi^{2}
$$

The forms (15) and (13) are equivalent. Solving for $\omega$ from the equation of motion of $\omega$ and inserting back into (15) is a valid manipulation. In any instance it is easy to check that (13) and (15) are totally equivalent.

Consider now the case when we keep the form (15), but where now $\omega$ is not a fundamental field, but rather is given in terms of a "Maximal Rank" Gauge Field, i.e.,

$$
\omega=\epsilon^{\mu \nu \alpha \beta} \partial_{[\mu} A_{\nu \alpha \beta]}
$$

$\partial_{[\mu} A_{\nu \alpha \beta]}$ being the "Maximal Rank" Gauge Field for the four dimensional case. We have now the invariance $A_{\nu \alpha \beta} \rightarrow A_{\nu \alpha \beta}+\partial_{[\nu} \Lambda_{\alpha \beta]}$. 
The equation of motion associated with the variation of $A_{\nu \alpha \beta}$ is now

$$
\epsilon^{\mu \nu \alpha \beta} \partial_{\beta}\left(\omega-\lambda^{\frac{1}{2}} \phi^{2}\right)=0
$$

which is integrated to

$$
\omega=\lambda^{\frac{1}{2}} \phi^{2}+M
$$

where $M$ is a space time constant that will be responsible for the s.s.b. of scale invariance. Once again, as in the point particle case, we can see the effect of $M$ by studying the other eqs. of motion, in this case, that of $\phi$,

$$
\partial_{\mu} \partial^{\mu} \phi=-2 \omega \lambda^{\frac{1}{2}} \phi
$$

and by using (18), we get,

$$
\partial_{\mu} \partial^{\mu} \phi=-2 \lambda \phi^{3}-2 M \lambda^{\frac{1}{2}} \phi=-\frac{\partial V_{e f f}}{\partial \phi}
$$

where

$$
V_{e f f}=\frac{1}{2} \lambda \phi^{4}+M \lambda^{\frac{1}{2}} \phi^{2}
$$

We see that the constant of integration $M$, which is associated to s.s.b. of scale invariance is responsible for spontaneous generation of mass $^{2}$ which

equals $2 M \lambda^{\frac{1}{2}}$, notice that if $M<0$, we obtain also s.s.b. of the $\phi \rightarrow-\phi$ reflection symmetry, because $\phi$ gets an expectation value.

\section{Spontaneous Breaking of Scale Symmetry in a Generally Covariant Model}

This section gives an example of work on scale invariant generally covariant theories. There is no attempt to go through all the work done in this area, but rather present one example where the "Maximal rank" gauge field produces the s.s.b. of s.i. in a generally covariant theory.

As opposed to the other sections of this paper, the material presented here has already appeared elswhere. This material is included nevertheless because it matches perfectly with the other sections and helps to complement and understand the general phenomenon studied here. 
In the first scale invariant model of this kind $^{7}$, a metric, a dilaton field and a "measure field" $\Phi$ are introduced. The unusual modified measure ${ }^{8}$ is an object that has the same transformation properties under general coordinate transformations as $\sqrt{-g} d^{4} x\left(g=\operatorname{det}\left(g_{\mu \nu}\right)\right)$. $\Phi$ is a density built out of degrees of freedom independent of the metric. For example, given a four index field strength (in four dimensions) $F_{\mu \nu \alpha \beta}=\partial_{[\mu} A_{\nu \alpha \beta]}$ as $\Phi=\epsilon^{\mu \nu \alpha \beta} F_{\mu \nu \alpha \beta}$.

Therefore we also allow in the action, in addition to the ordinary measure of integration $\sqrt{-g} d^{4} x$, another one, $\Phi d^{4} x$, where $\Phi$ is a density built out of degrees of freedom independent of the metric. One may consider the field strength to be composed of elementary scalars ${ }^{9}$. Four in the case of a four index field strength. For example, given 4-scalars $\varphi_{a}(\mathrm{a}=1,2,3,4)$, one can construct the density

$$
\Phi=\varepsilon^{\mu \nu \alpha \beta} \varepsilon_{a b c d} \partial_{[\mu} \varphi_{a} \partial_{\nu} \varphi_{b} \partial_{\alpha} \varphi_{c} \partial_{\beta]} \varphi_{d}
$$

so that

$$
A_{\nu \alpha \beta}=\varepsilon_{a b c d} \varphi_{a} \partial_{\nu} \varphi_{b} \partial_{\alpha} \varphi_{c} \partial_{\beta} \varphi_{d}
$$

The modified measure idea has been applied to dynamical generation of string and brane tensions ${ }^{10}$, to cosmological ${ }^{11}$ and to the fermion family problem $^{12}$

One can allow both geometrical measures to enter the theory and consider

$$
S=\int L_{1} \Phi d^{4} x+\int L_{2} \sqrt{-g} d^{4} x
$$

Here $L_{1}$ and $L_{2}$ are $\varphi_{a}$ independent. There is a good reason not to consider mixing of $\Phi$ and $\sqrt{-g}$, like for example using $\frac{\Phi^{2}}{\sqrt{-g}}$. This is because there is then an invariance (up to the integral of a total divergence) under the infinite dimensional symmetry $\varphi_{a} \rightarrow \varphi_{a}+f_{a}\left(L_{1}\right)$ where $f_{a}\left(L_{1}\right)$ is an arbitrary function of $L_{1}$ if $L_{1}$ and $L_{2}$ are $\varphi_{a}$ independent. Such symmetry (up to the integral of a total divergence) is absent if mixed terms are present. A $\frac{\Phi^{2}}{\sqrt{-g}}$ can be included in the action, it is consistent with scale invariance, but would lead to a non zero vacuum energy density in the ground state of the theory.

We will study now the dynamics of a scalar field $\phi$ interacting with gravity as given by the action (24) with

$$
L_{1}=\frac{-1}{\kappa} R(\Gamma, g)+\frac{1}{2} g^{\mu \nu} \partial_{\mu} \phi \partial_{\nu} \phi-V(\phi), L_{2}=U(\phi)
$$


$R(\Gamma, g)=g^{\mu \nu} R_{\mu \nu}(\Gamma), R_{\mu \nu}(\Gamma)=R_{\mu \nu \lambda}^{\lambda}, R_{\mu \nu \sigma}^{\lambda}(\Gamma)=\Gamma_{\mu \nu, \sigma}^{\lambda}-\Gamma_{\mu \sigma, \nu}^{\lambda}+\Gamma_{\alpha \sigma}^{\lambda} \Gamma_{\mu \nu}^{\alpha}-\Gamma_{\alpha \nu}^{\lambda} \Gamma_{\mu \sigma}^{\alpha}$.

In the variational principle $\Gamma_{\mu \nu}^{\lambda}, g_{\mu \nu}$, the measure fields scalars $\varphi_{a}$ and the scalar field $\phi$ are all to be treated as independent variables.

If we perform the global scale transformation $(\theta=$ constant $)$

$$
g_{\mu \nu} \rightarrow e^{\theta} g_{\mu \nu}
$$

then the action, is invariant provided $V(\phi)$ and $U(\phi)$ are of the form

$$
V(\phi)=f_{1} e^{\alpha \phi}, U(\phi)=f_{2} e^{2 \alpha \phi}
$$

and $\varphi_{a}$ is transformed according to $\varphi_{a} \rightarrow \lambda_{a} \varphi_{a}$ (no sum on a) which means $\Phi \rightarrow\left(\prod_{a} \lambda_{a}\right) \Phi \equiv \lambda \Phi$ such that $\lambda=e^{\theta}$ and $\phi \rightarrow \phi-\frac{\theta}{\alpha}$. In this case we call the scalar field $\phi$ needed to implement scale invariance "dilaton".

Let us consider the equations which are obtained from the variation of the $\varphi_{a}$ fields. We obtain then $A_{a}^{\mu} \partial_{\mu} L_{1}=0$ where $A_{a}^{\mu}=\varepsilon^{\mu \nu \alpha \beta} \varepsilon_{a b c d} \partial_{\nu} \varphi_{b} \partial_{\alpha} \varphi_{c} \partial_{\beta} \varphi_{d}$. Since det $\left(A_{a}^{\mu}\right)=\frac{4^{-4}}{4 !} \Phi^{3} \neq 0$ if $\Phi \neq 0$. Therefore if $\Phi \neq 0$ we obtain that $\partial_{\mu} L_{1}=0$, or that $L_{1}=M$, where $\mathrm{M}$ is constant.

If the three index field potential is elementary rather than being a composite of primitive fields $\varphi_{a}$, then still the equation $L_{1}=M$, where $\mathrm{M}$ is constant is obtained.

This constant $\mathrm{M}$ appears in a self-consistency condition of the equations of motion that allows us to solve for $\chi \equiv \frac{\Phi}{\sqrt{-g}}$ (for details see Ref.7.)

$$
\chi=\frac{2 U(\phi)}{M+V(\phi)} \text {. }
$$

To get the physical content of the theory, it is convenient to go to the Einstein conformal frame where

$$
\bar{g}_{\mu \nu}=\chi g_{\mu \nu}
$$

In terms of $\bar{g}_{\mu \nu}$ the non Riemannian contribution (defined as $\Sigma_{\mu \nu}^{\lambda}=\Gamma_{\mu \nu}^{\lambda}-\left\{\begin{array}{l}\lambda \\ \mu \nu\end{array}\right\}$ where $\left\{\begin{array}{l}\lambda \\ \mu \nu\end{array}\right\}$ is the Christoffel symbol), disappears from the equations, which can be written then in the Einstein form $\left(R_{\mu \nu}\left(\bar{g}_{\alpha \beta}\right)=\right.$ usual Ricci tensor $)$

$$
R_{\mu \nu}\left(\bar{g}_{\alpha \beta}\right)-\frac{1}{2} \bar{g}_{\mu \nu} R\left(\bar{g}_{\alpha \beta}\right)=\frac{\kappa}{2} T_{\mu \nu}^{e f f}(\phi)
$$


where

$$
T_{\mu \nu}^{e f f}(\phi)=\phi_{, \mu} \phi_{, \nu}-\frac{1}{2} \bar{g}_{\mu \nu} \phi_{, \alpha} \phi_{, \beta} \bar{g}^{\alpha \beta}+\bar{g}_{\mu \nu} V_{e f f}(\phi), V_{e f f}(\phi)=\frac{1}{4 U(\phi)}(V+M)^{2} .
$$

If $V(\phi)=f_{1} e^{\alpha \phi}$ and $U(\phi)=f_{2}$ as required by scale invariance, we obtain from the above equation,

$$
V_{e f f}=\frac{1}{4 f_{2}}\left(f_{1}+M e^{-\alpha \phi}\right)^{2}
$$

Also a minimum is achieved at zero cosmological constant for the case $\frac{f_{1}}{M}<0$ at the point $\phi_{\min }=\frac{-1}{\alpha} \ln \left|\frac{f_{1}}{M}\right|$. Finally, the second derivative of the potential $V_{\text {eff }}$ at the minimum is $V_{\text {eff }}^{\prime \prime}=\frac{\alpha^{2}}{2 f_{2}}\left|f_{1}\right|^{2}>0$ if $f_{2}>0$, so that a realistic scalar field potential, with massive excitations when considering the true vacuum state, is achieved in a way consistent with the idea of scale invariance. Since we can always perform the transformation $\phi \rightarrow-\phi$ we can choose by convention $\alpha>0$. We then see that as $\phi \rightarrow \infty, V_{\text {eff }} \rightarrow \frac{f_{1}^{2}}{4 f_{2}}=$ const. providing an infinite flat region.

The constant $M$ gives, once again s.s.b. of scale invariance. The conserved currents and the reasons why when $M \neq 0$ these do not lead to a conserved charge have been studied in Ref. 15. This explains also the absence of a Goldstone boson in spite of having s.s.b. of s.i..

\section{Spontaneous Generation of Confining Be- havior}

Let us now turn our attention to gauge theories and consider first the standard pure Yang Mills action

$$
S=\int d^{4} x L, L=-\frac{1}{4} F_{\mu \nu}^{a} F^{a \mu \nu}
$$

where

$$
F_{\mu \nu}^{a}=\partial_{\mu} A_{\nu}^{a}-\partial_{\nu} A_{\mu}^{a}+g f^{a b c} A_{\mu}^{b} A_{\nu}^{c}
$$

This theory is invariant under the scale symmetry

$$
A_{\mu}^{a}(x) \rightarrow A_{\mu}^{a}(x)^{\prime}=\lambda A_{\mu}^{a}(\lambda x)
$$


Let us rewrite (34) with the help of an auxiliary field $\omega$

$$
S=\int d^{4} x L, L=\frac{1}{4} \omega^{2}-\frac{1}{2} \omega \sqrt{F_{\mu \nu}^{a} F^{a \mu \nu}}
$$

upon solving the equation of motion obtained from the variation of $\omega$ and then replacing into the action (37) we get back (34).

Let us consider now the replacement $\omega \rightarrow \epsilon^{\mu \nu \alpha \beta} \partial_{[\mu} A_{\nu \alpha \beta]}$

and consider now the equation of motion obtained from the variation of $A_{\nu \alpha \beta}$, which is

$$
\epsilon^{\gamma \delta \alpha \beta} \partial_{\beta}\left(\omega-\sqrt{F_{\mu \nu}^{a} F^{a \mu \nu}}\right)=0
$$

which is solved by

$$
\omega=\sqrt{F_{\mu \nu}^{a} F^{a \mu \nu}}+M
$$

$M$ being once again a space-time constant which produces s.s.b. of s.i. and in this case it is furthermore associated with the spontaneous generation of confining behavior. Indeed the equations of motion obtained from (37) in the case $\omega$ is replaced by $\epsilon^{\mu \nu \alpha \beta} \partial_{[\mu} A_{\nu \alpha \beta]}$, have the form,

$$
\nabla_{\mu}\left(\left(\sqrt{F_{\alpha \beta}^{b} F^{b \alpha \beta}}+M\right) \frac{F^{a \mu \nu}}{\sqrt{F_{\alpha \beta}^{b} F^{b \alpha \beta}}}\right)=0
$$

If we consider the case the argument of the square root is positive, the square root itself is defined as positive and $M>0$ as well, then, it is not the case that $\sqrt{F_{\alpha \beta}^{b} F^{b \alpha \beta}}$ aquires an expectation value. In this case, in the limit of weak field strengths (which dominates the infrared behavior) eq. (40) gives

$$
\nabla_{\mu}\left(\frac{F^{a \mu \nu}}{\sqrt{F_{\alpha \beta}^{b} F^{b \alpha \beta}}}\right)=0
$$

Such an equation of motion describe a confining theory ${ }^{16,17}$. For example among other properties, it is known that electric monopoles do not exist ${ }^{17}$. Also string like solutions are a remarkable consequence of such equation ${ }^{16,17}$.

\section{Discussion}

There are several sources for the possible presence of a maximal rank field strength. One possibility is of course is that they are provided from the begining from a microscopic theory. Eleven dimensional supergravity contains 
four index field strengths ${ }^{18}$ and although four index field strengths are not maximal rank field strengths in eleven dimensions, they are maximal rank field strengths of the effective four dimensional theory (in the low energy limit).

Another way in which maximal rank field strengths could appear could be a totally different mechanism: infrared rather than microscopic. Let us consider the gauge theory example. There the actions (34) and (37) are totally equivalent. In (37), the introduction of the $\omega$ field is like the introduction of intermediate states between two operators, so that $A^{2} \rightarrow \Sigma A|n\rangle\langle n| A$, where $\mid n>$ is a complete set of states.

In the same way, the introduction of the $\omega$ field and the $\omega$ field terms in (37) give rise to a gaussian integral in the functional integral. The result of doing the functional integral gives us back the action (34). Thinking of the $\omega$ field as the functional analog of the states $|n\rangle$, we see that with the help of this field, we are writing $F_{\mu \nu}^{a} F^{a \mu \nu}$ as the product of $\sqrt{F_{\mu \nu}^{a} F^{a \mu \nu}} \times \sqrt{F_{\mu \nu}^{a} F^{a \mu \nu}}$ and we insert then the intermediate states, represented by $\omega$.

The replacement

$$
\omega \rightarrow \epsilon^{\mu \nu \alpha \beta} \partial_{[\mu} A_{\nu \alpha \beta]}
$$

has then a simple interpretation. As we see from the result of such replacement, the connection between $\omega$ and the gauge field incorporates now the arbitrary constant $M$ as we can see from eq. (39). That is the zero energy momentum component of $\omega$ becomes undetermined. Since it is well known that the nonabelian gauge theory has severe infrared divergences, which are associated with undetermined chromomagnetic field strengths ${ }^{19,20}$, declaring this "ignorance" on the zero energy momentum part of the "intermediate states" represented by $\omega$ appears to be a well motivated step. A proposal for the classification of the phases of a gauge theory, including the confinement phase, has been discussed in terms of the zero energy momentum gauge field content of the vacuum ${ }^{21}$ and flux tube confinement effects due to the zero energy momentum gluons in the vacuum have also been proposed ${ }^{22}$. The constant $M$ could have the interpretation then of the contribution of the zero energy momentum gluons in the vacuum which are responsible for confinement. 


\section{Acknowledgments}

I would like to thank A.Kaganovich for discussions.

\section{References}

[1] C.G. Callan, Jr., S. Coleman and R. Jackiw, Ann. Phys. (N.Y.) 59, 42 (1970).

[2] S. Coleman and R. Jackiw, Ann. Phys. (N.Y.) 67, 552 (1971).

[3] For a review see R. Jackiw, "Investigations in current algebra", in "Current Algebra and anomalies", S.B.Treitman, R. Jackiw, B.Zumino and E.Witten, World Scientific, 1985, Singapore.

[4] A. Zee, Phys. Rev. Lett. 42, 417 (1979).

[5] S. Adler, Phys. Rev. Lett. 44, 1567 (1980), Phys. Lett. 95B, 241 (1980); K.Akama, Y. Chikashige, T. Matsuki and H.Terrazawa, Prog. Theo. Phys. 60, 1900 (1980).

[6] These ideas are a realization of the "elasticity of the vacuum" of A. Sakharov, Dokl. Nauk. 177, 70 (1967).

[7] E.I. Guendelman, Mod. Phys. Lett. A14, 1043 (1999); Mod. Phys. Lett. A14, 1397, (1999); gr-qc/9901067.

[8] For a review on non scale invariant versions of these models see, E.I.Guendelman and A.B.Kaganovich, Phys.Rev.D.60, 065004 (1999). Early papers on the subject: E.I.Guendelman and A.B.Kaganovich, Phys.Rev.D.53, 7020 (1996); Phys.Rev.D.55, 5970 (1997).

[9] E.I. Guendelman, E.Nissimov and S.Pacheva, Phys. Lett. 360B, 57 (1995); hep-th/9903245; C.Castro, Int. J. Mod. Phys. A13, 1263 (1998).

[10] E.I. Guendelman, Class. Quantum Grav.17, 3673 (2000); E.I. Guendelman, Phys. Rev. D63: 046006 (2001); E.I. Guendelman, A. Kaganovich, E. Nissimov and S. Pacheva, Phys. Rev. D66: 046003 (2002); hepth/0304269; hep-th/0210062. 
[11] E.I. Guendelman and O.Katz, Class. Quantum Grav. 20, 1715 (2003) and references there.

[12] E.I.Guendelman and A.B.Kaganovich, Int. J. Mod. Phys. A17, 417 (2002); Mod. Phys. Lett. A17, 1227 (2002); Int. J. Mod. Phys. A17, 4419 (2002); Int. J. Mod. Phys. D11, 1595 (2002).

[13] A.Aurilia, H.Nicolai and P.K.Townsend, Nucl. Phys. B176, 509 (1980); S.W.Hawking, Phys. Lett. 134B, 258 (1984); A.Aurilia, G.Dernardo, F.Legovini and E.Spallucci, Phys. Lett. 147B, 258 (1984); E.Witten, in Sheter II, 1985, Proc. of 1983 Sheter Island Conference on Quantum Field Theory and the Fundamental Problems of Physics (Cambridge MA, MIT press); J.D.Brown and C.Teitelboim, Phys. Lett. 195B, 177 (1987); Nucl. Phys. B297, 787 (1988).

[14] E.I.Guendelman and J.D.Bekenstein, Phys. Rev. D35, 716 (1987).

[15] E.I.Guendelman, Class. Quantum Grav. 17, 361 (2000).

[16] A.Aurilia, A.Smailagic and E. Spallucci, Phys. Rev. D47, 2536 (1993) and references.

[17] E.I.Guendelman, Phys. Lett. 412B, 42 (1997); N.Amer and E.I.Guendelman, Int. J. Mod. Phys. A15, 4407 (2000).

[18] For a review see, M.J.Duff, "The World in eleven dimensions: supergravity, supermembranes and M-theory" (IOP Publishing, 1999).

[19] G.K.Savvidy, Phys. Lett. 71B, 133 (1977); N.K.Nielsen and P.Olesen, Nucl. Phys. B144, 376 (1978).

[20] In (non scale invariant) three dimensional gauge theories the need to introduce zero energy gauge fields is already apparent in perturbation theory. See for example E.I.Guendelman and Z.M.Radulovich, Phys. Rev. D27, 357 (1983); Phys. Rev. D30, 1338 (1984); E.I.Guendelman, Int. J. Mod. Phys. A5, 4341 (1990). The infrared divergences of these theories were studied first by R.Jackiw and S. Templeton, Phys. Rev. D23, 2291 (1981); S. Templeton, Phys. Lett. 103B, 134 (1981) and T.Appelquist and R.Pisarski, Phys. Rev. D23, 2305 (1981). 
[21] See for example, E.I.Guendelman, Int. J. Mod. Phys. A5, 2783 (1990).

[22] E.I.Guendelman and D.A.Owen, Mod. Phys. Lett. A6, 41 (1991). For a review of other works concerning the possible relation of non trivial gauge field strengths in the vacuum and confinement see "The quark confinement model of hadrons", eds. G.V.Efimov and M.A.Ivanov (IOP Publishing, 1993). 